\title{
RENEWAL CHARACTERIZATION OF MARKOV MODULATED POISSON PROCESSES $\dagger$
}

\author{
MARCEL F. NEUTS \\ Department of Systems and Industrial Engineering \\ University of Arizona \\ Tucson, Arizona \\ USHIO SUMITA \\ William E. Simon Graduate School of Business Administration \\ University of Rochester \\ Rochester, NY 14627 \\ and \\ YOSHITAKA TAKAHASHI \\ NTT Communication Switching Laboratories \\ Musashino-shi, Tokyo 180, Japan
}

\begin{abstract}
A Markov Modulated Poisson Process (MMPP) M(t) defined on a Markov chain $J(t)$ is a pure jump process where jumps of $M(t)$ occur according to a Poisson process with intensity $\lambda_{i}$ whenever the Markov chain $J(t)$ is in state $i$. $\mathrm{M}(\mathrm{t})$ is called strongly renewal $(S R)$ if $\mathrm{M}(\mathrm{t})$ is a renewal process for an arbitrary initial probability vector of $\mathrm{J}(\mathrm{t})$ with full support on $P=\left\{\mathrm{i}: \lambda_{\mathrm{i}}>0\right\} . \quad \mathrm{M}(\mathrm{t})$ is called weakly renewal $(W R)$ if there exists an initial probability vector of $\mathrm{J}(\mathrm{t})$ such that the resulting MMPP is a renewal process. The purpose of this paper is to develop general characterization theorems for the class $S R$ and some sufficiency theorems for the class $W R$ in terms of the first passage times of the bivariate Markov chain $[J(t), M(t)]$. Relevance to the lumpability of $J(t)$ is also studied.
\end{abstract}

Key Words: Markov modulated Poisson processes, transition rate matrix, first passage times, renewal processes, lumpability

AMS subject classification index: $60 \mathrm{~K} 05,60 \mathrm{~K} 15,90 \mathrm{C} 40$

\section{$\S 0$ INTRODUCTION}

Let $\mathrm{J}(\mathrm{t})$ be a Markov chain on $N=\{0,1,2, \cdots, \mathrm{N}\}$ governed by a transition rate matrix $\underline{\underline{v}}=\left[v_{\mathrm{ij}}\right]$. Let $v_{\mathrm{i}}=\sum_{\mathrm{j} \in N} v_{\mathrm{ij}}$. The infinitesimal generator $\underline{\underline{Q}}$ is then given by

† This paper has been partially supported by the IBM program of Support for Education in the Management of Information Systems. 
$\underline{Q}=-\underline{\underline{v}}_{D}+\underline{\underline{v}}$ where $\underline{\underline{v}}_{D}=\operatorname{diag}\left\{v_{\mathrm{i}}\right\}$ is the diagonal matrix of size $\mathrm{N}+1$ whose $\mathrm{i}$-th diagonal element is $v_{i}$. A Markov modulated Poisson process $M(t)$ governed by $J(t)$ and a nonnegative vector $\underline{\lambda}^{T}=\left[\lambda_{0}, \cdots, \lambda_{N}\right]$ is a pure jump process where jumps of $M(t)$ occur according to a Poisson process with intensity $\lambda_{\mathrm{i}}$ whenever the Markov chain $\mathrm{J}(\mathrm{t})$ is in state i. Following Neuts (1988), we say that the Markov modulated Poisson process, hereafter denoted by MMPP, has a source $\left(\underline{Q}, \underline{\underline{\Lambda}}_{D}\right)$ where $\underline{\underline{\Lambda}}_{D}=\operatorname{diag}\left\{\lambda_{\mathrm{i}}\right\}$. For convenience, we define

(0.1) $P=\left\{\mathrm{i}: \lambda_{\mathrm{i}}>0, \mathrm{i} \in N\right\} ; Z=\left\{\mathrm{i}: \lambda_{\mathrm{i}}=0, \mathrm{i} \in N\right\}$.

To avoid triviality, we assume throughout the paper that $\mathrm{J}(\mathrm{t})$ is irreducible and hence ergodic.

In general, the interarrival times generated from an MMPP are not independent nor identically distributed. In many applications of telecommunication systems, it is known that interarrival times of voice/data packets to a communication channel are correlated and do not constitute a renewal process. For such applications, an MMPP is often appropriate for describing an input stream to the system. See, e.g., Heffes and Lucantoni (1986) and Sriram and Whitt (1986). A single server queueing system with an $\mathrm{N}$-process input stream and general i.i.d. service times has been studied by Ramaswami (1980), where an MMPP is a special case of N-process. Subsequently, Burman and Smith (1986) and Knessl and Matkowsky (1987) have studied a single server queueing system with an MMPP input stream and general i.i.d. service times with more specific details. These results facilitate the use of MMPP's in applications. The reader is also referred to Neuts (1988) for a comprehensive discussion of the MMPP.

The question of under what conditions an MMPP becomes a renewal process is a classical one. A stationary doubly stochastic Poisson process is a generalization of the Poisson process where the intensity $\lambda(t)$ is a nonnegative-valued stochastic process. It is clear that an MMPP with a source $\left(\underline{\underline{Q}}, \underline{\underline{\Lambda}}_{D}\right)$ is a stationary doubly stochastic Poisson process having the intensity $\lambda(\mathrm{J}(\mathrm{t}))$. Kingman (1964) has shown that a stationary doubly stochastic process is a renewal process if and only if its rate function takes on the values $\lambda$ $>0$ and zero alternatively on the successive intervals of a stationary alternating renewal process and the intervals where the rate $\lambda$ prevails have an exponential distribution. These conditions are described more explicitly for specific MMPP's by Meier (1984) and Neuts (1988).

A source $\left(\underline{Q}, \underline{\underline{\Lambda}}_{\mathrm{D}}\right)$ of MMPP's may produce a renewal process only for specific initial distributions of the underlying Markov chain $\mathrm{J}(\mathrm{t})$. Some other sources of MMPP's yield a renewal process for any initial distribution with support on $P$. More formal definitions are now introduced.

Definition 0.1 A source $\left(\underline{\underline{Q}}, \underline{\underline{\Lambda}}_{\mathrm{D}}\right)$ of MMPP's is called strongly renewal, denoted by $\left(\underline{Q}, \underline{\underline{\Lambda}}_{\mathrm{D}}\right) \in S R$, if for any initial distribution of $\mathrm{J}(\mathrm{t})$ with full support on $P$ the resulting MMPP is a renewal process.

Definition 0.2 A source ( $\left.\underline{\underline{Q}}, \underline{\underline{\Lambda}}_{\mathrm{D}}\right)$ of MMPP's is called weakly renewal, denoted by 
$\left.\underline{Q}, \underline{\underline{\Lambda}}_{\mathrm{D}}\right) \in W R$, if there exists an initial distribution $\pi_{0}^{\mathrm{T}}$ of $\mathrm{J}(\mathrm{t})$ such that the resulting MMPP is a renewal process.

It is clear that $S R \subset W R$. For convenience, we also say that an MMPP with source ( $\underline{\mathrm{Q}}$, $\underline{\underline{\Lambda}}_{\mathrm{D}}$ ) is strongly renewal (weakly renewal) if the MMPP is a renewal process with ( $\left.\underline{\underline{Q}}, \underline{\underline{\Lambda}}_{\mathrm{D}}\right)$ $\in S R\left(\left(\underline{\underline{Q}}, \underline{\underline{\Lambda}}_{\mathrm{D}}\right) \in W R\right)$.

The purpose of this paper is to develop general characterization theorems for the class $S R$ and some sufficiency theorems for the class $W R$ in terms of the first passage times of the bivariate Markov chain [ $J(t), M(t)]$. Relevance to the lumpability of $J(t)$ is also studied. In Section 1, we analyze the first passage times of $[J(t), M(t)]$, which provides a basic tool for subsequent study. A necessary and sufficient condition is given in Section 2 for the class $S R$. It is shown that a source $\left(\underline{Q}, \underline{\underline{\Lambda}}_{\mathrm{D}}\right)$ belongs to $S R$ if and only if the first passage time of the bivariate process $[\mathrm{J}(\mathrm{t}), \mathrm{M}(\mathrm{t})]$ from (i,m) to $\{(\mathrm{j}, \mathrm{m}+1): \mathrm{j} \in N\}$ is equal in distribution for all $i \in P$. As an immediate corollary, we show that if jumps of an MMPP are triggered at only one state, say $i \in N$, then the MMPP is a renewal process. The necessary and sufficient condition is then expressed more explicitly in terms of a transition rate matrix of the underlying Markov chain. The relevance of the lumpability of $\mathrm{J}(\mathrm{t})$ to the class $S R$ is discussed in Section 3. It is shown that if $\mathrm{J}(\mathrm{t})$ is lumpable with respect to lumping $\{P, Z\}$ and $\lambda_{\mathrm{i}}=\lambda$ for all $\mathrm{i} \in P$, then the associated MMPP is strongly renewal, but the converse is not true. When the lumpability of $J(t)$ is present, the interarrival time distribution for jumps of $M(t)$ can be evaluated explicitly in the real domain. Section 4 is devoted to the study of the class $W R$. A sufficient condition is given under which an MMPP belongs to WR. This sufficient condition can be reinterpreted in terms of a transition rate matrix of the underlying Markov chain. For illustrating differences between the two classes $W R$ and $S R$, an example is given where an MMPP which belongs to $W R$ but not to $S R$ is constructed explicitly. Also given is a characterization theorem for an MMPP to be a Poisson process. Finally in Section 5, some concluding remarks are given.

\section{$\S 1$ FIRST PASSAGE TIMES OF BIVARIATE MARKOV CHAIN [J(t), M(t)]}

Let $M(t)$ be an MMPP as described in Section 0 , having a source $\left(\underline{Q}, \underline{\underline{\Lambda}}_{D}\right)$. We recall that the infinitesimal generator $\underline{Q}$ of the underlying Markov chain $J(t)$ has a form

$$
\underline{\underline{Q}}=-\underline{\underline{v}} D+\underline{\underline{v}} .
$$

One easily sees that the bivariate process $[\mathrm{J}(\mathrm{t}), \mathbf{M}(\mathrm{t})]$ is Markov. Figure 1.1 illustrates typical transitions governing $[\mathrm{J}(\mathrm{t}), \mathrm{M}(\mathrm{t})]$.

It should be noted that the bivariate process $[\mathrm{J}(\mathrm{t}), \mathrm{M}(\mathrm{t})$ ] is spatially homogeneous columnwise. Furthermore, the marginal process $M(t)$ is a pure jump process. Consequently, the first passage time of $[J(t), M(t)]$ from $(i, m)$ to any state in the $(m+1)$-th column does not depend on $\mathrm{m}$. We define

$$
\mathrm{T}_{\mathrm{i}}^{+}=\inf \{\mathrm{t}:[\mathrm{J}(\mathrm{t}), \mathrm{M}(\mathrm{t})] \in(\mathrm{j}, \mathrm{m}+1) \text { for some } \mathrm{j} \in N \quad \mid[\mathrm{J}(0), \mathrm{M}(0)] \in(\mathrm{i}, \mathrm{m})\}
$$

and 


$$
\phi_{i}^{+}(s)=E\left[e^{-s T_{i}^{+}}\right]
$$

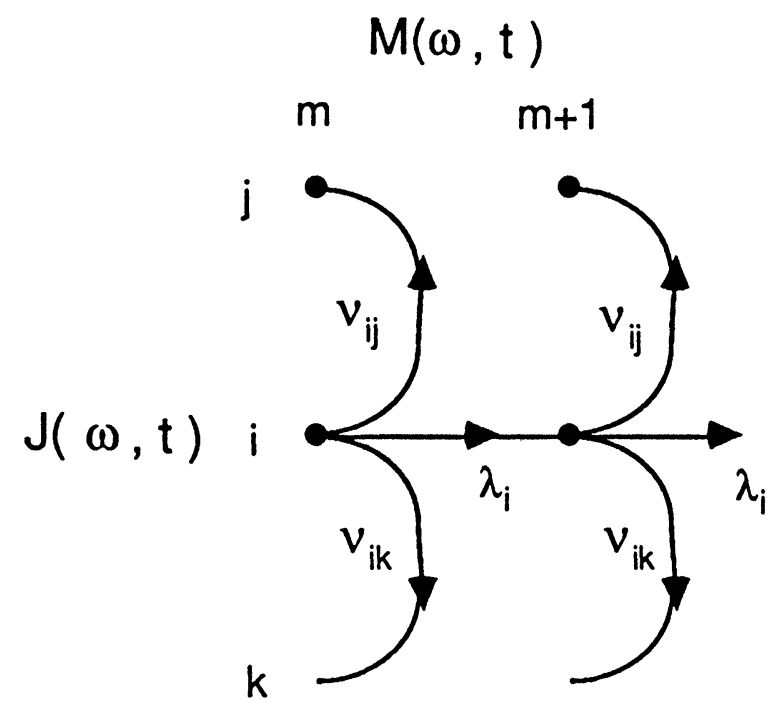

Figure 1.1 Typical Transitions of $[J(t), M(t)]$

For convenience, we introduce a random variable $\mathrm{V}_{\mathrm{i}}$ on $N$ where $\mathrm{V}_{\mathrm{i}}=\mathrm{j}$ means that the bivariate process $[\mathrm{J}(\mathrm{t}), \mathrm{M}(\mathrm{t})]$ enters the $(\mathrm{m}+1)$-th column for the first time at $\mathrm{j} \in N$ given that it starts at $i \in N$ in the $\mathrm{m}$-th column. Correspondingly, we define

$$
\underline{\xi}^{+}(s)=\left[\xi_{\mathrm{ij}}^{+}(\mathrm{s})\right] ; \xi_{\mathrm{ij}}^{+}(\mathrm{s})=\int_{0}^{\infty} \mathrm{e}^{-s \mathrm{sx}} \mathrm{P}\left[\mathrm{T}_{\mathrm{i}}^{+} \leq \mathrm{dx}, \mathrm{V}_{\mathrm{i}}=\mathrm{j}\right] .
$$

Let $\phi^{+}(s)=\left[\phi_{0}^{+}(s), \cdots, \phi_{N}^{+}(s)\right]^{T}$. It then follows from (1.2) through (1.4) that

$$
\Phi^{+}(\mathrm{s})=\underline{\xi}^{+}(\mathrm{s}) \underline{\mathrm{e}}
$$

where $\underline{e}$ is a column vector with all components equal to 1 .

To evaluate $\underline{\xi}^{+}(s)$, we consider a bivariate process $\left[\mathrm{J}^{*}(\mathrm{t}), \mathrm{M}^{*}(\mathrm{t})\right]$ on $S=\{(\mathrm{n}, \mathrm{m}): \mathrm{n} \in N, \mathrm{~m}=0,1\}$ governed by the transition rate matrix $\underline{\underline{v}}^{*}$ where

$$
\underline{\underline{v}}^{*}=\left(\begin{array}{cc}
\underline{\underline{v}} & \underline{\underline{\Lambda}} \mathrm{D} \\
\underline{\underline{0}} & \underline{\underline{0}}
\end{array}\right)
$$

Here the first block corresponds to $\mathrm{G}=\{(\mathrm{n}, 0): \mathrm{n} \in N\}$ and the second block to $\mathrm{B}=\{(\mathrm{n}, 1)$ $: \mathrm{n} \in N$ \}. It should be noted that states in $\mathrm{B}$ are absorbing. If we define 


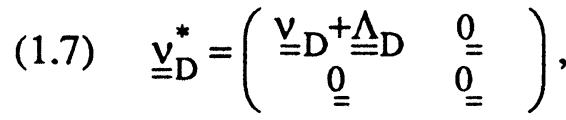

the infinitesimal generator $\underline{Q}^{*}$ governing $\left[J^{*}(t), M^{*}(t)\right]$ is given by

(1.8) $\quad \underline{Q}^{*}=-\underline{\underline{v}}_{\mathrm{D}}^{*}+\underline{\underline{v}}^{*}$.

Let $\underline{\underline{P}}^{*}(t)=\left[p_{(n, m)\left(n^{\prime}, m^{\prime}\right)}^{*}(t)\right]$ be the transition probability matrix of $\left[J^{*}(t), M^{*}(t)\right]$,

and define $\underline{\pi}^{*}(s)=\int_{0}^{\infty} \mathrm{e}^{-s t} \underline{\underline{P}}^{*}(\mathrm{t}) \mathrm{dt}$. Since $\frac{\mathrm{d}}{\mathrm{dt}} \stackrel{\underline{P}}{ }^{*}(\mathrm{t})=\underline{\underline{P}}^{*}(\mathrm{t}) \underline{\underline{Q}}^{*}$ and $\underline{\underline{P}}^{*}(0)=\underline{\underline{I}}$, one has $\underline{\underline{\pi}}^{*}(\mathrm{~s})$ $=\left[s \underline{\underline{I}}-\underline{\underline{Q}}^{*}\right]^{-1}$. It then follows from (1.6) through (1.8) that

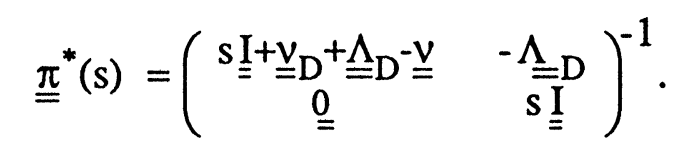

One easily sees that for the matrix $\underline{\underline{X}}$ of the form

$$
\underline{\underline{X}}=\left(\begin{array}{ll}
\underline{\underline{a}} & \underline{\bar{b}} \\
\underline{\underline{0}} & \underline{\underline{I}}
\end{array}\right),
$$

with a being nonsingular, the inverse $\underline{\underline{X}}^{-1}$ is given by

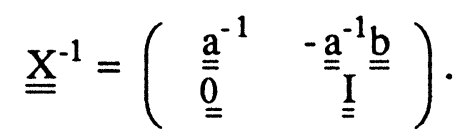

If we define

$$
\underline{\underline{\theta}}_{D}(s)=\operatorname{diag}\left\{\left(s+v_{i}+\lambda_{i}\right)^{-1}\right\}_{i \in N},
$$

it follows from (1.9) and (1.11) that

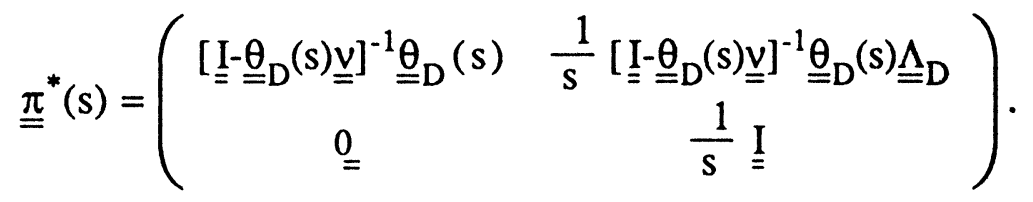

We are now in a position to prove the following theorem.

Theorem 1.1 Let $\underline{\xi}^{+}(\mathrm{s})$ and $\Phi^{+}(\mathrm{s})$ be as defined in (1.4) and (1.5). Then

(a) $\quad \underline{\xi}^{+}(\mathrm{s})=\left[\underline{\underline{\mathrm{I}}}-\underline{\underline{\theta}}_{\mathrm{D}}(\mathrm{s}) \underline{\underline{\underline{v}}}\right]^{-1} \underline{\underline{\theta}}_{\mathrm{D}}(\mathrm{s}) \underline{\underline{\Lambda}}_{\mathrm{D}}$ and 
(b) $\Phi^{+}(s)=\left[\underline{\underline{I}}-\underline{\underline{\theta}}_{D}(s) \underline{\underline{v}}\right]^{-1} \underline{\underline{\theta}}_{D}(s) \underline{\lambda}$.

\section{Proof.}

From the construction of $\left[\mathrm{J}^{*}(\mathrm{t}), \mathrm{M}^{*}(\mathrm{t})\right]$, one easily sees that $P\left[T_{i}^{+} \leq t, V_{i}=j\right]=p^{*}{ }_{(i, 0)(j, 1)}(t)$. Hence from $(1.4)$, one has $\xi_{i j}^{+}(s)=s \pi^{*}{ }_{(i, 0)(j, 1)}(s)$. Statement (a) then follows from (1.13). Part (b) is immediate from (1.5), and the theorem is proved.

We note that $\xi_{\mathrm{ij}}^{+}(\mathrm{s})=0$ for $\mathrm{j} \in Z$. This corresponds to the fact that $\{(\mathrm{j}, \mathrm{m}+1)$ : $\mathrm{j} \in N\}$ can be reached from $\{(\mathrm{j}, \mathrm{m}): \mathrm{j} \in N\}$ only through $\{(\mathrm{i}, \mathrm{m}): \mathrm{i} \in P\}$. It is also worth noting that Theorem 1.1 (b) yields an interesting recursion formula regarding $\phi_{i}^{+}(\mathrm{s})$. One sees that

$$
\Phi^{+}(s)=\underline{\underline{\theta}}_{D}(s) \underline{\lambda}+\underline{\underline{\theta}}_{D}(s) \underline{\underline{v}} \Phi^{+}(s) .
$$

This equation can be rewritten componentwise as

$$
\phi_{i}^{+}(s)=\left(\frac{\lambda_{i}}{v_{i}+\lambda_{i}}\right)\left(\frac{v_{i}+\lambda_{i}}{s+v_{i}+\lambda_{i}}\right)+\left(\frac{v_{i}}{v_{i}+\lambda_{i}}\right)\left(\frac{v_{i}+\lambda_{i}}{s+v_{i}+\lambda_{i}}\right) \sum_{k \in N} \frac{v_{i k}}{v_{i}} \phi_{k}^{+}(s) .
$$

The probabilistic interpretation of (1.14) is now clear. Suppose $\lambda_{i}>0$. The sojourn time of $[J(t), M(t)]$ at state $(i, m)$ is exponentially distributed with parameter $v_{i}+\lambda_{i}$. Upon expiration of this sojourn time, the process immediately moves up to $(\mathrm{i}, \mathrm{m}+1)$ with probability $\lambda_{\mathrm{i}} /\left(v_{\mathrm{i}}+\lambda_{\mathrm{i}}\right)$. With probability $\left(v_{\mathrm{i}} /\left(v_{\mathrm{i}}+\lambda_{\mathrm{i}}\right)\right)\left(v_{\mathrm{ik}} / \nu_{\mathrm{i}}\right)$, the state transition is to $(\mathrm{k}, \mathrm{m})$. In this case, it takes further $\mathrm{T}_{\mathrm{k}}^{+}$for the process to reach $\{(\mathrm{i}, \mathrm{m}+1): \mathrm{i} \in N\}$. The case $\lambda_{\mathrm{i}}=$ 0 can be interpreted similarly.

\section{§2 CHARACTERIZATION OF STRONGLY RENEWAL MMPP'S}

In this section, we develop a necessary and sufficient condition under which an MMPP becomes a renewal process for any initial distribution of $\mathrm{J}(\mathrm{t})$ with full support on $P$. Simpler necessary or sufficient conditions are also discussed. Let $\left(\tau_{\mathrm{k}}\right)_{\mathrm{k}=0}^{\infty}$ be a sequence of jump epochs of the marginal process $M(t)$ where $\tau_{0}=0$. We define

(2.1) $X_{k}=\tau_{k}-\tau_{k-1}, k=1,2,3 \ldots$.

Of interest are the joint probability functions characterized by the vector transform

$$
\zeta_{\mathrm{K}}(\underline{s})=\int_{0} \mathrm{e}^{-\mathrm{s}^{\mathrm{T}} \underline{\mathrm{X}}} \mathrm{P}\left[\mathrm{X}_{1} \leq \mathrm{dx}_{1}, \mathrm{X}_{2} \leq \mathrm{dx}_{2}, \ldots, \mathrm{X}_{\mathrm{K}} \leq \mathrm{dx}_{\mathrm{K}}\right]
$$

where $\underline{\mathrm{s}}=\left[\mathrm{s}_{1}, \mathrm{~s}_{2}, \ldots, \mathrm{s}_{\mathrm{K}}\right]^{\mathrm{T}}$ and $\underline{\mathrm{X}}=\left[\mathrm{X}_{1}, \mathrm{X}_{2}, \ldots, \mathrm{X}_{\mathrm{K}}\right]^{\mathrm{T}}$. For convenience, we also define 


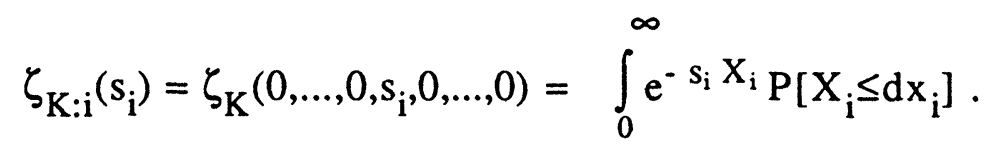

From the columnwise spatial homogeneity of the bivariate process $[\mathrm{J}(\mathrm{t}), \mathrm{M}(\mathrm{t})]$, one has

$$
\zeta_{K}(s)=\pi_{0}^{T} \prod_{k=1}^{K} \xi^{+}\left(s_{k}\right) e
$$

where $\underline{\xi}^{+}(s)$ is defined in (1.4) and $\pi_{0}^{T}$ is the initial distribution of $J(t)$. Equation (2.4) can be found in Neuts (1988).

We next derive the main result of this section. The fact that $\mathrm{X}$ and $\mathrm{Y}$ are equal in distribution is denoted by $\mathrm{X} \stackrel{\mathrm{d}}{=} \mathrm{Y}$. For index sets $\mathrm{A}, \mathrm{B} \subset N$, a submatrix of $\underline{\underline{a}}=\left[\mathrm{a}_{\mathrm{ij}}\right]$ onto $A \times B$ is denoted by $\underset{=A B}{a}=\left[a_{i j}\right]_{i \in A, j \in B}$.

Theorem 2.1 Let $\mathrm{M}(\mathrm{t})$ be an MMPP having a source $\left(\underline{\underline{\mathrm{Q}}}, \underline{\underline{\Lambda}}_{\mathrm{D}}\right)$. Let $\mathrm{J}(0) \in P$ with probability one. Then $M(t)$ is strongly renewal if and only if $\mathrm{T}_{i}^{+} \stackrel{d}{=} \mathrm{T}_{j}^{+}$for all $i, j \in P$. Furthermore, the Laplace transform of i.i.d. interarrival times is given by

$$
\eta(s)=\underline{u}_{i}^{T}\left[\underline{\underline{I}}-\underline{\underline{\theta}}_{D}(s) \underline{\underline{v}}\right]^{-1} \underline{\underline{\theta}}_{D}(s) \underline{\lambda},
$$

where $\underline{u}_{i}^{\mathrm{T}}$ is the $\mathrm{i}$-th unit vector for some $\mathrm{i} \in P$.

Proof.

First note from the definition of $P$ that

$$
\underline{\underline{\Lambda}}_{\mathrm{D}}=\left(\begin{array}{cc}
\underline{\underline{\Lambda}}_{\mathrm{D}: \mathrm{PP}} & \underline{\underline{ }} \mathrm{PZ} \\
\underline{\underline{0} \mathrm{ZP}} & \underline{0} \\
\mathrm{ZZZ}
\end{array}\right)
$$

From Theorem 1.1 (a), this then implies that

$$
\underline{\xi}^{+}(s)=\left(\begin{array}{ll}
\underline{\xi}_{P P}^{+}(s) & \underline{0}_{P Z} \\
\underline{\xi}_{Z P}^{+}(s) & \underline{0}_{Z Z}
\end{array}\right)
$$

By the assumption that $\mathrm{J}(0) \in P$ with probability one, the initial distribution $\pi_{0}^{\mathrm{T}}$ is of the form $\pi_{0}^{\mathrm{T}}=\left[\pi_{0: \mathrm{P}}^{\mathrm{T}}, \varrho_{Z}^{\mathrm{T}}\right]$. From (2.4) and (2.6), one concludes that 


$$
\zeta_{K}(\underline{s})=\pi_{0: P}^{T} \prod_{k=1}^{K} \underline{\xi}_{P P}^{+}\left(s_{k}\right) \underline{e}_{P}
$$

It should be noted from (2.3) that

$$
\zeta_{K: i}\left(s_{i}\right)=\pi_{0: P}^{T} \prod_{k=1}^{i-1} \xi_{P P}^{+}(0+) \underline{\xi}_{P P}^{+}\left(s_{i}\right) \underline{e}_{P},
$$

since $\underline{\xi}^{+}(0+)$ is a stochastic matrix from (1.4) and $\underline{\xi}_{\mathrm{PP}}^{+}(0+) \underline{\mathrm{e}}_{\mathrm{P}}=\underline{\mathrm{e}}_{\mathrm{P}}$ from (2.6).

Suppose $\mathrm{T}_{\mathrm{i}}^{+} \stackrel{\mathrm{d}}{=} \mathrm{T}_{\mathrm{j}}^{+}$for all $\mathrm{i}, \mathrm{j} \in P$ so that $\phi_{\mathrm{P}}^{+}(\mathrm{s})=\eta(\mathrm{s}) \mathbf{e}_{\mathrm{P}}$ from Theorem $1.1(\mathrm{~b})$.

Since $\Phi^{+}(s)=\underline{\xi}^{+}(s) \underline{e}$, this together with (2.6) implies that $\underline{\xi}_{\mathrm{PP}}^{+}(\mathrm{s}) \underline{\mathrm{e}}_{\mathrm{P}}=\eta(\mathrm{s}) \mathrm{e}_{\mathrm{P}}$.

It then follows from (2.7) that

$$
\zeta_{K}(s)=\prod_{i=1}^{K} \eta\left(s_{i}\right)
$$

Consequently, $X_{i}(1 \leq i \leq K)$ are i.i.d. random variables having $\eta(s)=E\left[e^{-s X i}\right]$. Since $\pi_{0: P}^{\mathrm{T}}$ is chosen arbitrarily, $M(t)$ is strongly renewal.

Conversely, suppose $M(t)$ is strongly renewal. One then has $\zeta_{\mathrm{K}: 1}(\mathrm{~s})=\zeta_{\mathrm{K}: 2}(\mathrm{~s})$. From (2.8), this in turn implies that

$$
\pi_{0: P}^{T} \underline{\xi}_{P P}^{+}(s) \underline{e}_{P}=\pi_{0: P}^{T} \underline{\xi}_{P P}^{+}(0+) \underline{\xi}_{P P}^{+}(s) \underline{e}_{P} .
$$

Since $\pi_{0: P}^{T}$ can be chosen as an arbitrary probability vector on $P$, it follows with $\Phi_{\mathrm{P}}^{+}(\mathrm{s})=$ $\underline{\xi}_{\mathrm{PP}}^{+}(\mathrm{s}) \mathrm{e}_{\mathrm{P}}$ that

$$
\Phi_{\mathrm{P}}^{+}(\mathrm{s})=\underline{\underline{\xi}}_{\mathrm{PP}}^{+}(0+) \Phi_{\mathrm{P}}^{+}(\mathrm{s}) .
$$

$\Phi_{\mathrm{P}}^{+}(\mathrm{s})$ is a right eigenvector of a stochastic matrix $\underline{\xi}_{\mathrm{PP}}^{+}(0+)$ with eigenvalue 1 for all s with $\operatorname{Re}(s)>0$. Hence $\Phi_{\mathrm{P}}^{+}(s)=\eta(s) \underline{\mathrm{e}}_{\mathrm{P}}$, and the proof is completed.

The necessary and sufficient condition of Theorem 2.1 holds trivially when jumps of $M(t)$ can be triggered only through one state, say, $i \in N$. In other words, $\lambda_{i}>0$ and $\lambda_{\mathrm{j}}=0$ for all $\mathrm{j} \in N \backslash\{\mathrm{i}\}$, so that $P$ is a singleton set $P=\{\mathrm{i}\}$. The next corollary is then immediate. 
Corollary 2.2 If $P$ is a singleton set, say, $P=\{\mathrm{i}\}$ and $\mathrm{J}(0)=\mathrm{i}$ with probability one, then $\mathrm{M}(\mathrm{t})$ is strongly renewal.

It can be readily seen that if a and $\underline{\underline{d}}$ are nonsingular, one has

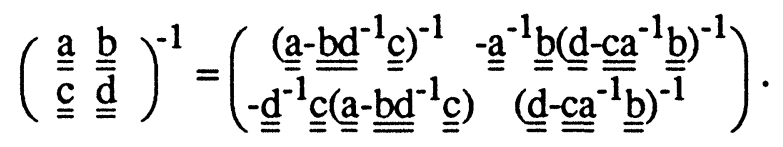

Since $\Phi^{+}(s)=\underline{\xi}^{+}(s)$ e, it then follows from Theorem 1.1 (a) and (2.11) that

$$
\Phi_{\mathrm{P}}^{+}(\mathrm{s})=\left[\mathrm{sI}_{\mathrm{PP}}+\underline{\underline{v}}_{\mathrm{D}: \mathrm{PP}}+\underline{\underline{\Lambda}}_{\mathrm{D}: \mathrm{PP}}-\underline{\underline{v}}_{\mathrm{PP}}-\underline{\underline{\mathrm{v}}}_{\mathrm{PZ}} \underline{\underline{Z}}_{\mathrm{ZP}}(\mathrm{s})\right]^{-1} \underline{\lambda}_{\mathrm{P}},
$$

where

(2.13) $\quad \underline{\beta}_{Z P}(s)=\left(s_{\underline{Z}} I+\underline{v}_{D}: Z Z-\underline{v}_{Z Z}\right)^{-1} \underline{\underline{v}}_{Z P}$

and

(2.14) $\Phi_{Z}^{+}(s)=\underline{\beta}_{Z P}(s) \Phi_{P}^{+}(s)$.

The necessary and sufficient condition of Theorem 2.1 can now be expressed in a more explicit form.

Theorem 2.3 Let $\mathrm{M}(\mathrm{t})$ be an MMPP having a source $\left(\underline{\mathrm{Q}}, \underline{\underline{\Lambda}}_{\mathrm{D}}\right)$. Let $\mathrm{J}(0) \in P$ with probability one. Then $\mathrm{M}(\mathrm{t})$ is strongly renewal if and only if

(i) $\underline{\lambda}_{\mathrm{P}}=\lambda \underline{\mathrm{e}}_{\mathrm{P}}$;

(ii) $\underline{v}_{\mathrm{PZ}} \underline{\mathrm{e}}_{\mathrm{Z}}=\gamma \mathrm{e}_{\mathrm{P}}$; and

(iii) $\underline{\underline{v}}_{\mathrm{PZ}} \underline{\beta}_{Z P}(s) \underline{e}_{\mathrm{P}}=\rho(s) \underline{e}_{\mathrm{P}}$.

Proof.

Suppose $M(t)$ is strongly renewal so that $\Phi_{P}^{+}(s)=\eta(s) \underline{e}_{p}$. Substituting this into (2.12), one finds that

$$
\underline{\lambda}_{\mathrm{P}}=\left[\mathrm{s} \underline{\underline{I}}_{\mathrm{PP}}+\underline{\underline{v}}_{\mathrm{D}: \mathrm{PP}}+\underline{\underline{\Lambda}}_{\mathrm{D}: \mathrm{PP}}-\underline{\underline{v}}_{\mathrm{PP}}-\underline{\underline{v}}_{\mathrm{PZ}} \underline{\underline{Z}}_{\mathrm{ZP}}(\mathrm{s})\right] \eta(\mathrm{s}) \underline{\mathrm{e}}_{\mathrm{P}},
$$

which leads to

$$
\text { (2.15) } \underline{\lambda}_{\mathrm{P}}=\eta(\mathrm{s})\left[\underline{\mathrm{e}}_{\mathrm{P}}+\underline{\underline{v}}_{\mathrm{PZ}} \underline{\mathrm{e}}_{\mathrm{Z}}+\underline{\lambda}_{\mathrm{P}}-\underline{\underline{v}}_{\mathrm{PZ}} \underline{\underline{\beta}}_{\mathrm{ZP}}(\mathrm{s}) \underline{\mathrm{e}}_{\mathrm{P}}\right]
$$

where $\left(\underline{v}_{D: P P}-\underline{v}_{P P}\right) \underline{e}_{P}=\underline{v}_{P Z} \underline{e}_{Z}$ is employed. By letting $s \rightarrow \infty$ in (2.15), one finds that

(2.16) $\underline{\lambda}_{\mathrm{P}}=\lim _{\mathrm{s} \rightarrow \infty} \mathrm{s} \eta(\mathrm{s}) \underline{\mathrm{e}}_{\mathrm{P}}=\lambda \underline{\mathrm{e}}_{\mathrm{P}}$

and (i) follows. Substituting (2.16) into (2.15) yields

(2.17) $\underline{\underline{v}}_{\mathrm{PZ}}\left[\underline{\mathrm{e}}_{\mathrm{Z}}-\underline{\beta}_{\mathrm{ZP}}(\mathrm{s}) \underline{\mathrm{e}}_{\mathrm{P}}\right]=\tilde{\rho}(\mathrm{s}) \underline{\mathrm{e}}_{\mathrm{P}}$

where 
(2.18)

$$
\tilde{\rho}(\mathrm{s})=\frac{\lambda-\eta(\mathrm{s})(\mathrm{s}+\lambda)}{\eta(\mathrm{s})} \text {. }
$$

Again by letting $s \rightarrow \infty$ in (2.17), one obtains

$$
\underline{v}_{P Z} \underline{e}_{Z}=\lim _{s \rightarrow \infty} \tilde{\rho}(s) \underline{e}_{P}=\gamma \underline{e}_{P},
$$

and (ii) is proved. Substitution of (2.18) back into (2.17) then leads to

$$
\underline{\underline{v}}_{\mathrm{PZ}} \underline{\underline{\beta}}_{\mathrm{ZP}}(\mathrm{s}) \underline{\mathrm{e}}_{\mathrm{P}}=[\gamma-\tilde{\rho}(\mathrm{s})] \underline{\mathrm{e}}_{\mathrm{P}} .
$$

Part (iii) now follows by one's letting $\rho(s)=\gamma-\tilde{\rho}(s)$.

Conversely, suppose (i),(ii) and (iii) hold. It can be seen that

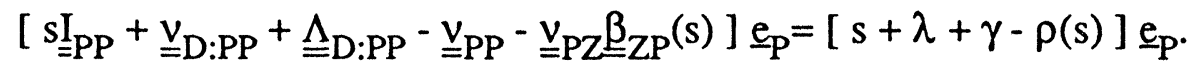

From (2.12) together with (2.20), one finds that

$$
\Phi_{\mathrm{P}}^{+}(\mathrm{s})=\lambda\left[\mathrm{sI}_{\mathrm{PP}}+\underline{\underline{v}}_{\mathrm{D}: \mathrm{PP}}+\underline{\underline{\Lambda}}_{\mathrm{D}: \mathrm{PP}}-\underline{\underline{v}}_{\mathrm{PP}}-\underline{\underline{v}}_{\mathrm{PZ}} \underline{\mathrm{B}}_{\mathrm{ZP}}(\mathrm{s})\right]^{-1} \underline{\mathrm{e}}_{\mathrm{P}}=\eta(\mathrm{s}) \underline{\mathrm{e}}_{\mathrm{P}}
$$

where

$$
\eta(s)=\frac{\lambda}{s+\lambda+\gamma-\rho(s)}
$$

and the theorem follows.

From (2.22), the next corollary is immediate.

Corollary 2.4 Let $M(t)$ be a strongly renewal MMPP. Then the Laplace transform $\eta(s)$ of the distribution of i.i.d. interarrival times is given by

$$
\eta(s)=\frac{\lambda}{s+\lambda+\gamma-\rho(s)}
$$

where $\lambda, \rho(s)$ and $\gamma$ are as given in (i),(ii), and (iii) of Theorem 2.3.

\section{\$3 RELEVANCE OF LUMPABILITY TO STONGLY RENEWAL MMPP'S}

In some applications, a Markov chain may be observed only through the passage of certain subsets of the state space and transitions within each subset are screened. The study of lumpability of Markov chains concerns the question of whether the resulting process is also a Markov chain, see e.g., Kemeny and Snell (1969). The analogous problem for semi-Markov processes has been studied by Cinlar $(1969,1971)$ and Serfozo (1971). In a recent paper by Sumita and Rieders (1987) it has been shown that a semi-Markov process is lumpable if and only if the first passage times $T_{i L(n)}$ and $T_{j L(n)}$ of the semi-Markov process are equal in distribution for all $i, j \in L(m)$ and all pairs of lumps $L(m)$ and $L(n)$. In the light of Kingman's theorem, one may then expect that the lumpability of the Markov chain $\mathrm{J}(\mathrm{t})$ implies the strongly renewal property of the associated MMPP. The purpose of this section is to formally study relevance of lumpability to strongly renewal MMPP's. 
Let $\mathrm{f}: N \rightarrow M$ be a function mapping $N=\{0,1, \ldots, \mathrm{N}\}$ onto $M=\{0,1, \ldots, \mathrm{M}\}$ where $0<\mathrm{M}<\mathrm{N}$. This function determines a partition $L=\{\mathrm{L}(0), \mathrm{L}(1), \ldots, \mathrm{L}(\mathrm{M})\}$ of $N$ where for $\mathrm{i} \in N$ and $\mathrm{k} \in M$,

(3.1) $\quad \mathrm{i} \in \mathrm{L}(\mathrm{k}) \Leftrightarrow \mathrm{f}(\mathrm{i})=\mathrm{k}$.

For $\mathrm{t} \geq 0$ let

(3.2) $\quad \mathrm{Y}(\mathrm{t}) \stackrel{\mathrm{d}}{=} \mathrm{f}(\mathrm{J}(\mathrm{t}))$.

Definition 3.1 A Markov chain $\mathrm{J}(\mathrm{t})$ is called lumpable with respect to a partition $L=$ $\{\mathrm{L}(0), \mathrm{L}(1), \ldots, \mathrm{L}(\mathrm{M})\}$, if for every initial state probability vector the process $\mathrm{Y}(\mathrm{t})$ determined by (3.2) is a Markov chain whose transition rate matrix is independent of the initial distribution. We denote this by $\mathrm{J}(\mathrm{t}) \in \operatorname{Lump}(L)$.

For a discrete time Markov chain $\mathrm{N}(\mathrm{k})$ governed by one-step transition probability

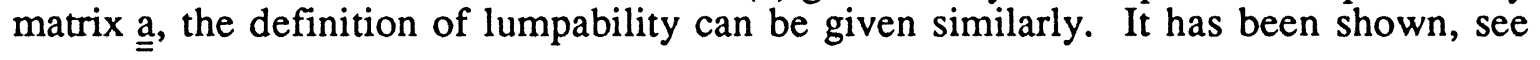
Kemeny and Snell (1969), that $\mathrm{N}(\mathrm{k}) \in \operatorname{Lump}(L)$ if and only if

\section{(3.3) $\quad \underline{\mathrm{VUaV}}=\underline{\mathrm{aV}}$.}

Here the matrices $\underline{\underline{V}}$ and $\underline{\underline{U}}$ are defined by

$$
\underline{\underline{\mathrm{V}}}=\left[\mathrm{v}_{\mathrm{im}}\right]_{\mathrm{i} \in N, \mathrm{~m} \in M} ; \mathrm{v}_{\mathrm{im}}=\left\{\begin{array}{l}
1 \text { if } \mathrm{i} \in \mathrm{L}(\mathrm{m}) \\
0 \text { if } \mathrm{i} \notin \mathrm{L}(\mathrm{m})
\end{array}\right.
$$

$$
\underline{\underline{U}}=\left[u_{\mathrm{mi}}\right]_{\mathrm{m} \in M, \mathrm{i} \in N} ; \mathrm{u}_{\mathrm{mi}}= \begin{cases}\frac{1}{|\mathrm{~L}(\mathrm{~m})|} & \text { if } \mathrm{i} \in \mathrm{L}(\mathrm{m}) \\ 0 & \text { if } \mathrm{i} \notin \mathrm{L}(\mathrm{m})\end{cases}
$$

where $|\mathrm{L}(\mathrm{m})|$ is the cardinality of $\mathrm{L}(\mathrm{m})$. For convenience, we say that a square matrix $\underline{\underline{b}}$ is lumpable with respect to $L$, denoted by $\underline{\underline{b}} \in \operatorname{Lump}(L)$ if $\underline{\underline{b}}$ satisfies (3.3).

The uniformization procedure of Keilson (1979) provides a bridge between continuous time and discrete time Markov chains in the following manner. For a continuous time Markov chain $J(t)$ governed by $\underline{\underline{v}}$, let $v>\max _{i}\left\{v_{i}\right\}$ and define

$$
\underline{a}=\underline{\underline{I}}-\frac{1}{v} \underline{v} D+\frac{1}{v} \underline{v} \text {. }
$$

Clearly $\stackrel{\mathrm{a}}{v}_{v}$ is a stochastic matrix and defines a discrete time Markov chain $\mathrm{N}(\mathrm{k})$. Let $\mathrm{K}_{\mathrm{v}}(\mathrm{t})$ be a Poisson process with intensity $v$. Then one has

$$
J(t)=N\left(K_{v}(t)\right)
$$

provided that the two processes share the same initial distribution. Sumita and Rieders (1987) have shown that $\mathrm{J}(\mathrm{t})$ is lumpable if and only if $\mathrm{N}(\mathrm{k})$ is lumpable. More specifically one has the following theorem:

Theorem 3.2 (Sumita and Rieders(1987))

$$
\mathrm{J}(\mathrm{t}) \in \operatorname{Lump}(L) \Leftrightarrow \underline{\underline{a}}_{\nu} \in \operatorname{Lump}(L) .
$$
MMPP's.

We are now in a position to study the relevance of lumpability to strongly renewal 
Theorem 3.3 Let $M(t)$ be an MMPP having a source $\left(\underline{Q}, \underline{\underline{\Lambda}}_{D}\right)$ and let $\underline{\xi}^{+}(\mathrm{s})$ be as in (1.4). Then the following three statements are equivalent.
(a) $\underline{\xi}^{+}(\mathrm{s}) \in \operatorname{Lump}(\{P, Z\})$.
(b) $\mathrm{J}(\mathrm{t}) \in \operatorname{Lump}(\{P, Z\})$ and $\underline{\lambda}_{\mathrm{P}}=\lambda \underline{\mathrm{e}}_{\mathrm{P}}$.
(c) $\underline{\underline{v}}_{\mathrm{PZ}} \underline{\mathrm{e}}_{\mathrm{Z}}=\gamma \underline{\mathrm{e}}_{\mathrm{P}}, \underline{\underline{v}}_{\mathrm{ZP}} \mathrm{e}_{\mathrm{P}}=\mu \underline{\mathrm{e}}_{\mathrm{Z}}$ and $\underline{\lambda}_{\mathrm{P}}=\lambda \underline{\underline{e}}_{\mathrm{P}}$.

\section{Proof.}

First show that (b) $\Leftrightarrow$ (c). From Theorem 3.2 and (3.6), it is clear that $\mathrm{J}(\mathrm{t}) \in \operatorname{Lump}(\{P, Z\})$ implies (c). Conversely suppose (c) holds. From (3.6), one has

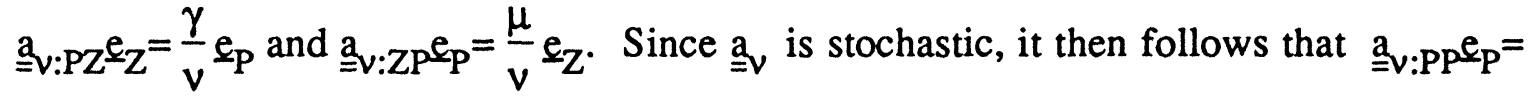

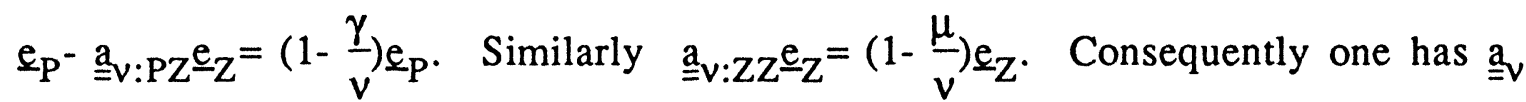
$\in \operatorname{Lump}(\{P, Z\})$, and (b) holds from Theorem 3.2.

Next prove (a) $\Leftrightarrow$ (c). Suppose $\underline{\xi}^{+}(\mathrm{s}) \in \operatorname{Lump}(\{P, Z\})$. Then from (2.6) and (3.3) there exist $\eta(s)$ and $\varepsilon(s)$ such that

$$
\Phi^{+}(s)=\underline{\xi}^{+}(s) \underline{e}=\left(\begin{array}{l}
\eta(s) \underline{e}_{\mathrm{p}} \\
\varepsilon(s) \underline{e}_{\mathrm{Z}}
\end{array}\right) .
$$

From Theorems 2.1 and 2.3, one has $\underline{\underline{v}}_{\mathrm{PZ}} \underline{\mathrm{e}}_{\mathrm{Z}}=\gamma \underline{\mathrm{e}}_{\mathrm{P}}$ and $\underline{\lambda}_{\mathrm{P}}=\lambda \underline{\mathrm{e}}_{\mathrm{P}}$. One also sees from (2.14) and (3.8) that

$$
\Phi_{Z}^{+}(s)=\eta(s) \underline{\beta}_{Z P}(s) \underline{e}_{P}=\varepsilon(s) \underline{e}_{Z} .
$$

It then follows from (2.13) that

i.e.,

$$
\begin{aligned}
\underline{\underline{v}}_{Z P} \underline{e}_{P} & =\frac{\varepsilon(s)}{\eta(s)}\left(s \underline{\underline{I}}_{Z Z}+\underline{\underline{v}}_{D: Z Z}-\underline{\underline{v}}_{Z Z}\right) \underline{e}_{Z} \\
& =\frac{\varepsilon(\mathrm{s})}{\eta(s)}\left(\mathrm{se}_{\mathrm{Z}}+\underline{\underline{v}}_{Z P} \underline{\mathrm{e}}_{\mathrm{P}}\right),
\end{aligned}
$$

$$
\underline{v}_{Z P} \underline{e}_{P}=\frac{\varepsilon(s)(s+\mu)}{\eta(s)} e_{Z}
$$

and (c) is proved. (See Remark 3.4 below.) that

Now suppose that (c) holds. It can be readily seen from Theorem 1.1 (a) and (3.6)

$$
\underline{\xi}^{+}(s)=\frac{1}{s+v+\lambda}[\underline{\underline{I}}-\underline{\underline{D}}(s)]^{-1} \underline{\underline{\Lambda}} \underline{D}
$$

where

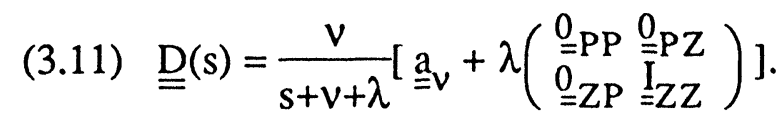

Since (b) and (c) are equivalent, one has $\underset{=}{\mathrm{a}} \in \operatorname{Lump}(\{P, Z\})$. This in turn implies from (3.11) that $\underline{\underline{D}}(s) \in \operatorname{Lump}(\{P, Z\})$. It has been shown in Lemma 1.4 of Sumita and Rieders 
(1987) that $(\underline{\underline{I}}-\triangleq)^{-1} \in \operatorname{Lump}(L)$ if and only if $\triangleq \in \operatorname{Lump}(L)$. Hence $\underline{\xi}^{+}(\mathrm{s}) \in \operatorname{Lump}(\{P$, $Z\})$, and the proof is completed.

Remark 3.4 It should be noted that the coefficient of $\underline{e}_{Z}$ in the right-hand side of (3.9), $\varepsilon(s)(s+\mu) / \eta(s)$, is equal to $\mu$ for all s such that $\operatorname{Re}(s)>0$. In fact, one has from Theorem 1.1 (a) that

$$
\underline{\underline{\theta}}_{D}(s)^{-1}\left[\underline{\underline{I}}-\underline{\underline{\theta}}_{D}(s) \underline{\underline{v}}\right] \phi^{+}(s)=\underline{\lambda} .
$$

This together with (3.8) implies that

(3.13) $-\eta(s) \underline{v}_{Z P} \underline{e}_{P}+\varepsilon(s)\left\{\underline{s}_{Z}+\underline{v}_{Z P} \underline{e}_{P}\right\}=\underline{0}_{Z}$.

Substituting the condition

(3.14) $\quad \underline{v}_{Z P} \underline{e}_{P}=\mu \underline{e}_{Z}$

into (3.13) yields

$$
\varepsilon(s)=\frac{\mu}{s+\mu} \eta(s)
$$

which gives for all $s$ with $\operatorname{Re}(\mathrm{s})>0$,

$$
\frac{\varepsilon(s)(s+\mu)}{\eta(s)}=\mu \text {. }
$$

The next corollary follows directly from Theorem 3.3.

Corollary 3.5 If $J(t) \in \operatorname{Lump}(\{P, Z\})$ (i.e., $\left.\underline{\underline{v}}_{\mathrm{PZ}} \underline{\mathrm{e}}_{\mathrm{Z}}=\gamma \underline{\mathrm{e}}_{\mathrm{P}}, \underline{\underline{v}}_{\mathrm{ZP}} \underline{\mathrm{e}}_{\mathrm{P}}=\mu \underline{\mathrm{e}}_{\mathrm{Z}}\right)$ and $\underline{\lambda}_{\mathrm{P}}=\lambda \underline{\mathrm{e}}_{\mathrm{P}}$, then $M(t)$ is strongly renewal.

We note that the difference between the conditions of Theorem 2.3 and those of Corollary 3.5 is that the former has

(3.15) $\quad \underline{v}_{P Z} \underline{\beta}_{Z P}(s) \underline{e}_{P}=\rho(s) \underline{e}_{P}$

while the latter requires (3.14). Though (3.14) implies (3.15), the converse is not true. The following example illustrates an MMPP which is strongly renewal but the underlying Markov chain is not lumpable with respect to $L=\{P, Z\}$.

Example 3.6 Let $N=\{0,1,2\}, P=\{0\}$ and $Z=\{1,2\}$. The underlying Markov chain $\mathrm{J}(\mathrm{t})$ is governed by

$$
\underline{\underline{v}}=\left(\begin{array}{c|cc}
0 & v_{01} & v_{02} \\
- & - & - \\
v_{10} & 0 & v_{12} \\
v_{20} & v_{21} & 0
\end{array}\right) .
$$

Let $\lambda_{0}=\lambda$, i.e., $\underline{\Lambda}_{\mathrm{D}}$ is of the form

$$
\Lambda_{\mathrm{D}}=\left(\begin{array}{c:ccc}
\lambda & 1 & 0 & 0 \\
\overline{-} & \bar{c} & \overline{0} \\
0 & 0 & 0 \\
0 & 1 & 0 & 0
\end{array}\right) \text {. }
$$

It follows from Corollary 2.2 that $\mathrm{M}(\mathrm{t})$ is strongly renewal, while $\mathrm{J}(\mathrm{t})$ is not lumpable with respect to $L=\{P, Z\}$, when $v_{10} \neq v_{20}$. 
When the underlying Markov chain is lumpable with respect to $L=\{P, Z\}$, the interarrival time distribution for the corresponding MMPP can be evaluated explicitly in the real domain as we show next.

Theorem 3.7 If $\mathrm{J}(\mathrm{t}) \in \operatorname{Lump}(\{P, Z\})$ and $\underline{\lambda}_{\mathrm{p}}=\lambda \underline{\mathrm{e}}_{\mathrm{p}}$, then the Laplace transform $\eta(\mathrm{s})$ of the interarrival time for the corresponding MMPP and its p.d.f. $f_{X}(t)$ are given by

(a)

$$
\begin{aligned}
\eta(s)= & \frac{\lambda(s+\mu)}{s^{2}+(\lambda+\mu+\gamma) s+\lambda \mu}, \\
f_{X}(t)= & \lambda \mu \cdot \frac{\exp \left[\frac{-H+\sqrt{H^{2}-4 \lambda \mu}}{2} \cdot t\right]-\exp \left[\frac{-H-\sqrt{H^{2}-4 \lambda \mu}}{2} \cdot t\right]}{\sqrt{H^{2}-4 \lambda \mu}} \\
& +\lambda \cdot \frac{\frac{-H+\sqrt{H^{2}-4 \lambda \mu}}{2} \exp \left[\frac{-H+\sqrt{H^{2}-4 \lambda \mu}}{2} \cdot t\right]}{\sqrt{H^{2}-4 \lambda \mu}} \\
& -\lambda \cdot \frac{\frac{-H-\sqrt{H^{2}-4 \lambda \mu}}{2} \exp \left[\frac{-H-\sqrt{H^{2}-4 \lambda \mu}}{2} \cdot t\right]}{\sqrt{H^{2}-4 \lambda \mu}},
\end{aligned}
$$

where $\lambda, \mu$ and $\gamma$ are as given in Theorem 3.3 (c) and $H=\lambda+\mu+\gamma$.

Proof.

Substituting $\underline{\underline{v}}_{P Z} \underline{e}_{Z}=\gamma \underline{e}_{P}$ and $\underline{\underline{v}}_{Z P} \underline{e}_{P}=\mu \underline{e}_{Z}$ into (3.15) yields

$$
\rho(s)=\gamma \frac{\mu}{s+\mu} \text {. }
$$

Then one has from Corollary 2.4 that

$$
\eta(s)=\frac{\lambda(s+\mu)}{s^{2}+(\lambda+\mu+\gamma) s+\lambda \mu},
$$

proving (a). Applying the Laplace inversion formula, one finds (b) from (a), completing the proof.

\section{§4 WEAKLY RENEWAL MMPP'S}

Even when an MMPP with source $\left(\underline{Q}, \underline{\underline{\Lambda}}_{D}\right)$ is not strongly renewal, an appropriate choice of an initial probability vector $\pi_{0: P}^{\mathrm{T}}$ on $P$ may still make the MMPP a renewal process, as we will see. The study of the class $W R$ of weakly renewal MMPP's necessitates certain subtlety and the characterization of $W R$ is much more difficult. The purpose of this section is to establish sufficient conditions under which an MMPP belongs to $W R$. Our first theorem is simple but useful.

Theorem 4.1 Let $\mathrm{M}(\mathrm{t})$ be an MMPP having a source $\left(\underline{\mathrm{Q}}, \underline{\underline{\Lambda}}_{\mathrm{D}}\right)$. If an initial probability vector $\pi_{0: P}^{\mathrm{T}}$ of $\mathrm{J}(\mathrm{t})$ on $P$ is chosen so that 
(4.1) $\pi_{0: P}^{\mathrm{T}} \underline{\xi}_{\mathrm{PP}}^{+}(\mathrm{s})=\chi(\mathrm{s}) \pi_{0: \mathrm{P}}^{\mathrm{T}}$, for all s with $\operatorname{Re}(\mathrm{s})>0$,

then $\mathrm{M}(\mathrm{t})$ belongs to $W R$. Furthermore the Laplace transform of the distribution of i.i.d. interarrival times is given by $\chi(\mathrm{s})$.

Proof.

Let $\zeta_{\mathrm{K}}(\mathrm{s})$ be as defined in (2.2). From (2.7) one then sees that

$$
\zeta_{\mathrm{K}}(\mathrm{s})=\underline{\pi}_{0: P}^{\mathrm{T}} \prod_{\mathrm{k}=1}^{\mathrm{K}} \underline{\xi}_{\mathrm{PP}}^{+}\left(\mathrm{s}_{\mathrm{k}}\right) \underline{\mathrm{e}}_{\mathrm{P}}=\prod_{\mathrm{k}=1}^{\mathrm{K}} \chi\left(\mathrm{s}_{\mathrm{k}}\right) \underline{\pi}_{0: P}^{\mathrm{T}} \mathrm{e}_{\mathrm{P}}=\prod_{\mathrm{k}=1}^{\mathrm{K}} \chi\left(\mathrm{s}_{\mathrm{k}}\right) \text {. }
$$

Hence the interarrival times $\mathrm{X}_{1}, \mathrm{X}_{2}, \ldots, \mathrm{X}_{\mathrm{K}}$ are i.i.d. random variables having $\chi(\mathrm{s})=$ $\mathrm{E}\left[\mathrm{e}^{-\mathrm{s} \mathrm{X}_{\mathrm{i}}}\right]$, and the theorem follows.

$W R$.

The following example illustrates that the class $S R$ is a proper subset of the class

Example 4.2 Let $\mathrm{J}(\mathrm{t})$ be a Markov chain on $N=\{0,1,2\}$ governed by transition rate matrix $\underline{\underline{ }}$ where

$$
\underline{\underline{v}}=\left(\begin{array}{cccc}
0 & 4 & 1 & 2 \\
1 & 0 & 1 & 5 \\
- & - & - & - \\
2 & 4 & 1 & 0
\end{array}\right)
$$

Let $P=\{0,1\}$ and $Z=\{2\}$ with $\lambda_{0}=\lambda_{1}=\lambda$ and $\lambda_{2}=0$. It should be noted that $\underline{\underline{P}}_{\mathrm{PZ}} \underline{\mathrm{e}}_{\mathrm{Z}}=$ $[2,5]^{\mathrm{T}}$ is not proportional to $\underline{\mathrm{e}}_{\mathrm{P}}=[1,1]^{\mathrm{T}}$, violating the condition of Theorem 2.3 (ii). Consequently the associated MMPP $M(t)$ is not strongly renewal. Let $\pi_{0: P}^{T}=[1 / 3,2 / 3]$.

From Theorem 1.1 and (2.12), one has

$$
\xi_{\mathrm{PP}}^{+}(s)^{-1}=\frac{1}{\lambda(s+6)}\left(\begin{array}{cc}
\mathrm{s}^{2}+(12+\lambda) s+32+6 \lambda & -4(s+8) \\
-(s+16) & \mathrm{s}^{2}+(12+\lambda) s+16+6 \lambda
\end{array}\right) \text {. }
$$

It then follows that $\pi_{0: P}^{\mathrm{T}} \underline{\xi}_{\mathrm{PP}}^{+}(\mathrm{s})^{-1}=1 / \chi(\mathrm{s}) \pi_{0: \mathrm{P}}^{\mathrm{T}}$ or equivalently $\pi_{0: \mathrm{P}}^{\mathrm{T}} \underline{\xi}_{\mathrm{PP}}^{+}(\mathrm{s})=\chi(\mathrm{s}) \pi_{0: \mathrm{P}}^{\mathrm{T}}$ where

(4.4) $\chi(s)=\frac{(s+6) \lambda}{s^{2}+(10+\lambda) s+6 \lambda}$.

Hence from Theorem $4.1, \mathrm{M}(\mathrm{t})$ belongs to $W R$. We note that the two zero points of the denominator of $\chi(\mathrm{s})$ in (4.4) sandwich -6 for any $\lambda>0$ and therefore $\chi(\mathrm{s})$ corresponds to a completely monotone density.

We next express the condition (4.1) of Theorem 4.1 in terms of transition rate matrix $\underline{\underline{v}}$ which is easier to examine.

Theorem 4.3 Let $M(t)$ be an MMPP having a source $\left(\underline{\underline{Q}}, \underline{\underline{\Lambda}}_{D}\right)$. Then the sufficient condition (4.1) for the MMPP to be weakly renewal with respect to $\pi_{0: P}^{\mathrm{T}}$ is equivalent to

(i) $\underline{\lambda}_{\mathrm{p}}=\lambda \underline{\mathrm{e}}_{\mathrm{P}}$; 
(ii) $\left.\quad \pi_{0: P}^{T} \underline{v}_{D: P P}-\underline{v}_{P P}\right)=\gamma \pi_{0: P}^{T} ;$ and

(iii) $\underline{\pi}_{0: P}^{T} \underline{v}_{P Z} \underline{\beta}_{Z P}(s)=\rho(s) \underline{\pi}_{0: P}^{T}$,

where $\underline{\beta}_{Z P}(s)$ is as in (2.13).

Proof.

First note from Theorem 1.1 and (2.11) that

$\underline{\xi}_{\mathrm{PP}}^{+}(\mathrm{s})=\left[\mathrm{sI}_{\mathrm{PP}}+\underline{\underline{v}}_{\mathrm{D}: \mathrm{PP}}+\underline{\underline{\Lambda}}_{\mathrm{D}: \mathrm{PP}}-\underline{\underline{v}}_{\mathrm{PP}}-\underline{\underline{v}}_{\mathrm{PZ}} \underline{\underline{\beta}}_{\mathrm{ZP}}(\mathrm{s})\right]^{-1} \underline{\underline{\Lambda}}_{\mathrm{D}: \mathrm{PP}}$

Suppose that the conditions (i),(ii) and (iii) hold true. One then sees that

$$
\begin{aligned}
\pi_{0: P}^{\mathrm{T}} \underline{\xi}_{\mathrm{PP}}^{+}(\mathrm{s})^{-1} & =\pi_{0: \mathrm{P}}^{\mathrm{T}} \underline{\Lambda}_{\mathrm{D}: \mathrm{PP}}{ }^{-1}\left[\underline{s}_{\mathrm{IPP}}+\underline{\underline{v}}_{\mathrm{D}: \mathrm{PP}}+\underline{\underline{\Lambda}}_{\mathrm{D}: \mathrm{PP}}-\underline{\underline{v}}_{\mathrm{PP}}-\underline{\underline{v}}_{\mathrm{PZ}} \underline{\mathrm{B}}_{\mathrm{ZP}}(\mathrm{s})\right] \\
& =1 / \chi(\mathrm{s}) \underline{\pi}_{0: \mathrm{P}}^{\mathrm{T}}
\end{aligned}
$$

where

(4.6)

$$
\chi(s)=\frac{\lambda}{s+\lambda+\gamma-\rho(s)}
$$

Hence the condition (4.1) holds.

Conversely suppose that the condition (4.1) holds true. Substituting (4.5) into (4.1) then yields

(4.7) $\pi_{0: \mathrm{P}}^{\mathrm{T}}=\chi(\mathrm{s}) \underline{\pi}_{0: \mathrm{P}}^{\mathrm{T}} \underline{\underline{D}}_{\mathrm{D}: \mathrm{PP}}{ }^{-1}\left[\underline{\mathrm{s}}_{\mathrm{PP}}+\underline{\underline{v}} \mathrm{D:PP}+\underline{\underline{\Lambda}}_{\mathrm{D}: \mathrm{PP}}-\underline{\underline{v}}_{\mathrm{PP}}-\underline{\underline{v}}_{\mathrm{PZ}} \underline{\underline{B}}_{\mathrm{ZP}}(\mathrm{s})\right]$.

By letting $s \rightarrow \infty$, one then can say

(4.8) $\pi_{0: \mathrm{P}}^{\mathrm{T}}=\lim _{\mathrm{s} \rightarrow \infty} \mathrm{s} \chi(\mathrm{s}) \pi_{0: \mathrm{P}}^{\mathrm{T}} \underline{\mathrm{D}}_{\mathrm{P} \mathrm{PP}}{ }^{-1}=\lambda \pi_{0: \mathrm{P}}^{\mathrm{T}} \underline{\Lambda}_{\mathrm{D}: \mathrm{PP}}{ }^{-1}$.

Hence $\underline{\lambda}_{\mathrm{P}}=\lambda \underline{\mathrm{e}}_{\mathrm{P}}$, and (i) is proved. To see (ii), substitute (i) into (4.7). One sees that (4.9) $\tilde{\rho}(\mathrm{s}) \underline{\pi}_{0: P}^{\mathrm{T}}=\underline{\pi}_{0: \mathrm{P}}^{\mathrm{T}}\left[\underline{\underline{v}}_{\mathrm{D}: \mathrm{PP}}-\underline{\underline{v}}_{\mathrm{PP}}-\underline{\underline{v}}_{\mathrm{PZ}} \underline{\underline{\beta}}_{\mathrm{ZP}}(\mathrm{s})\right]$

where

(4.10) $\tilde{\rho}(\mathrm{s})=\frac{\lambda-\chi(\mathrm{s})(\mathrm{s}+\lambda)}{\chi(\mathrm{s})}$.

Let

(4.11) $\gamma=\lim _{s \rightarrow \infty} \tilde{\rho}(s)$.

By letting $s \rightarrow \infty$ in (4.9), one can say that (ii) follows from (4.10) and (4.11). Substituting (ii) into (4.9), one finds that

(4.12) $\pi_{0: P}^{\mathrm{T}} \underline{\mathrm{v}}_{\mathrm{PZ}} \underline{\beta}_{Z \mathrm{P}}(\mathrm{s})=\rho(\mathrm{s}) \underline{\pi}_{0: \mathrm{P}}^{\mathrm{T}} ; \rho(\mathrm{s})=\gamma-\tilde{\rho}(\mathrm{s})$,

completing the proof.

Next corollary is immediate from (4.6).

Corollary 4.4 Let $M(t)$ be a weakly renewal MMPP satisfying (4.1). Then the Laplace transform $\chi(s)$ of the distribution of i.i.d. interarrival times is given by 


$$
\chi(s)=\frac{\lambda}{s+\lambda+\gamma-\rho(s)},
$$

where $\lambda, \rho(s)$ and $\gamma$ are as given in Theorem 4.3.

Before ending this section, we provide a characterization theorem for an MMPP to be a Poisson process.

Theorem 4.5 An MMPP M(t) is a Poisson process if and only if $\lambda_{i}=\lambda$ for all $i \in N$.

\section{Proof.}

First assume that an MMPP is a Poisson process with the arrival rate $\lambda$. Then there exists at least one probability vector $\pi_{0: P}^{\mathrm{T}}$ for which $M(t)$ is weakly renewal. From (4.6), one has $\chi(s)=\lambda /(s+\lambda+\gamma-\rho(s))=\lambda /(s+\lambda)$ so that $\gamma=\rho(s)$. It then follows from (4.12) that $\tilde{\rho}(\mathrm{s})=0$ and therefore $\gamma=0$ from (4.11). Hence $\rho$ (s) is also zero. Suppose $Z$ $\neq \varnothing$. Then the fact that $\rho(\mathrm{s})=0$ contradicts the condition of Theorem 4.3 (iii). Hence $Z=$ $\varnothing$.

Conversely suppose $Z=\varnothing$ and $\lambda_{i}=\lambda$ for all $i \in N$. Since $\underline{\lambda}_{P}=\lambda \underline{e}_{P}$ while the conditions of (ii) and (iii) of Theorem 2.3 are satisfied by default, $M(t)$ is strongly renewal. By setting $\gamma=\rho(s)=0$ in (2.22), one has $\eta(s)=\lambda /(s+\lambda)$, completing the proof.

\section{§5 CONCLUDING REMARKS}

It can be readily seen from (2.7) that an MMPP $M(t)$ is weakly renewal with respect to $\pi_{0: P}^{\mathrm{T}}$ if and only if there exists a Laplace transform $\eta(s)$ of a probability density function such that

$$
\zeta_{K}(s)=\pi_{0: P}^{T} \prod_{k=1}^{K} \xi_{P P}^{+}\left(s_{k}\right) e_{P}=\prod_{k=1}^{K} \eta\left(s_{k}\right)
$$

for all positive integers $\mathrm{K}$. In other words $\zeta_{\mathrm{K}}(\underline{\mathrm{s}})$ is interchangeable with respect to $\underline{\mathrm{s}}$ and decomposable as in (5.1). The characterization theorem for the class $S R$ could be obtained since $\pi_{0: P}^{T}$ could be chosen arbitrarily. However, a simple characterization of the class $W R$ is difficult. Some necessary conditions may be found if one could find conditions under which $\underline{P}_{\mathrm{P}}(\mathrm{s})=\underline{\xi}_{\mathrm{PP}}^{+}(\mathrm{s}) \underline{\mathrm{e}}_{\mathrm{P}}$ spans the $|P|$-dimensional Euclidian space by varying values of $\mathrm{s}$.

In this paper, only MMPP's having an initial distribution $\pi_{0: P}^{\mathrm{T}}$ with full support on $P$ have been considered for simplicity. Some of the results given in the paper can be easily extended to general MMPP's. If an MMPP $M(t)$ is strongly renewal, for example, $M(t)$ is always a delayed renewal process for an arbitrary initial distribution $\pi_{0}^{\mathrm{T}}$ on $N$. This type of extension for the class $W R$, however, is a little more subtle. 


\section{REFERENCES}

[1] Burman, D. Y. and Smith, D. R. (1986), "An Asymptotic Analysis of a Queueing System with Markov Modulated Arrival Process," Operat. Res., 34, 105-119.

[2] Cinlar, E. (1969), "Markov Renewal Theory," Adv. Appl. Prob., 1, 123-187.

[3] Cinlar, E. (1971), "Markov Renewal Theory: A Survey," Management Sci., 21, 727752.

[4] Heffes, H. and Lucantoni, D. M. (1986), "A Markov Modulated Characterization of Packetized Voice and Data Traffic and Related Statistical Multiplexer Performance," IEEE J. Selected Areas Commun., SAC-4, 6, 856-868.

[5] Keilson, J. (1979), Markov Chain Models ---Rarity and Exponentiality, SpringerVerlag.

[6] Kemeny, J. and Snell, L. (1969), Finite Markov Chains, Van Nostrand.

[7] Kingman, J. F. C. (1964), "On Doubly Stochastic Poisson Processes," Proc. Camb. Phil. Soc., 60, 923-930.

[8] Knessl, C. and Matkowsky, B. J. (1987), "A Markov Modulated M/G/1 Queue I: Stationary Distribution," Queueing Systems, 1, 355-374.

[10] Meier, K. S. (1984), "A Statistical Procedure for Fitting Markov-modulated Poisson Processes," Tech. Report No. 99B, University of Delaware.

[11] Neuts, M. F. (1988), Structured Stochastic Matrices of M/G/1 Type and Their Applications. (in preparation)

[12] Ramaswami, V. (1980), "The N/G/1 Queue and its Detailed Analysis," Adv. Appl. Prob., 12, 222-261.

[13] Serfozo, R. F. (1971), "Functions of Semi-Markov Processes," SIAM J. Appl. Math., 20, 3, 530-535.

[14] Sriram, K. and Whitt, W. (1986), "Characterizing Superposition Arrival Process in Packet Multiplexers for Voice and Data," IEEE J. Selected Areas Commun., SAC-4, 6, 833-846.

[15] Sumita, U. and Rieders, M. (1988), "First Passage Times and Lumpability of SemiMarkov Processes," J. Appl. Prob., 25, 675-687 


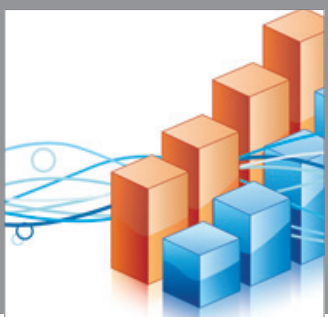

Advances in

Operations Research

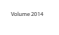

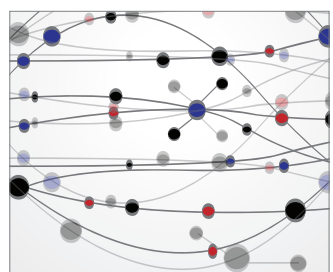

\section{The Scientific} World Journal
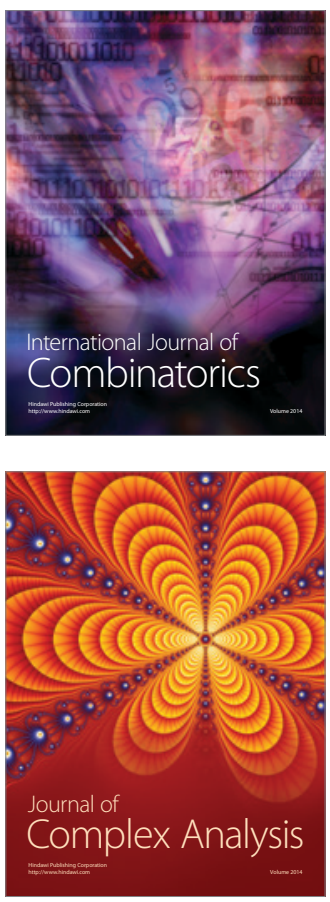

International Journal of

Mathematics and

Mathematical

Sciences
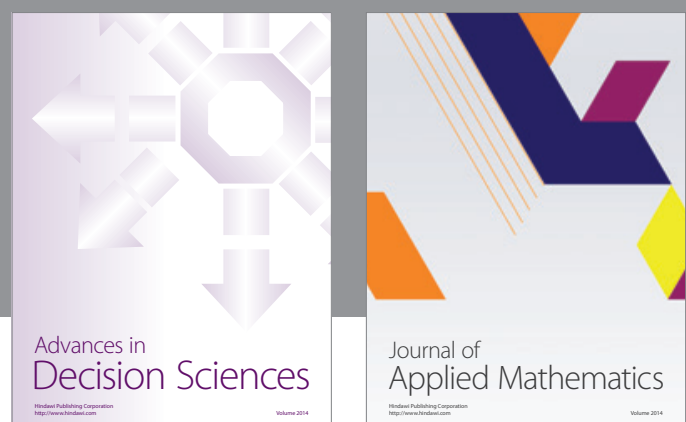

Journal of

Applied Mathematics
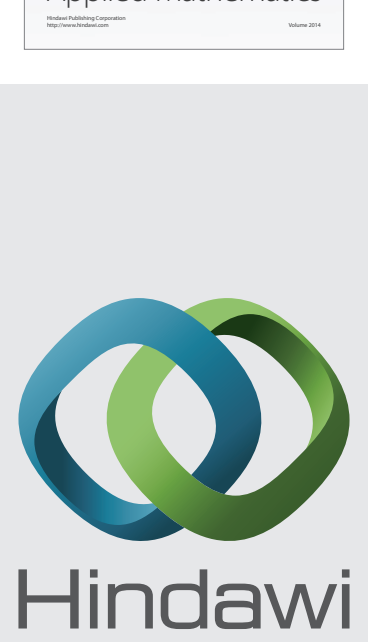

Submit your manuscripts at http://www.hindawi.com
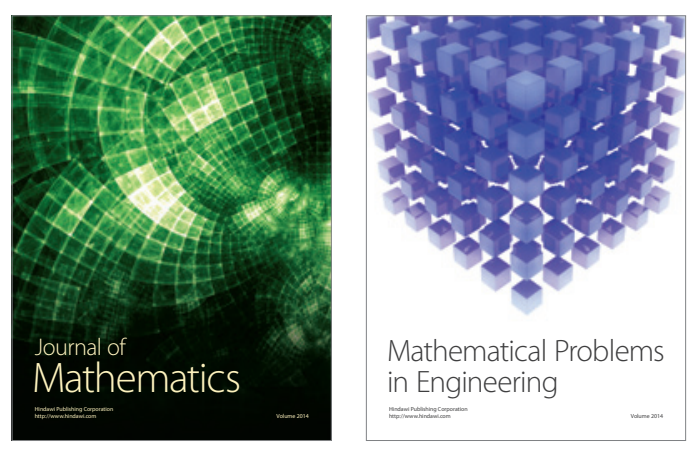

Mathematical Problems in Engineering
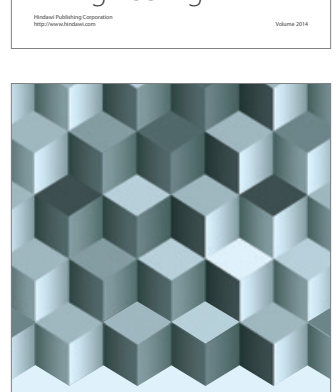

Journal of

Function Spaces
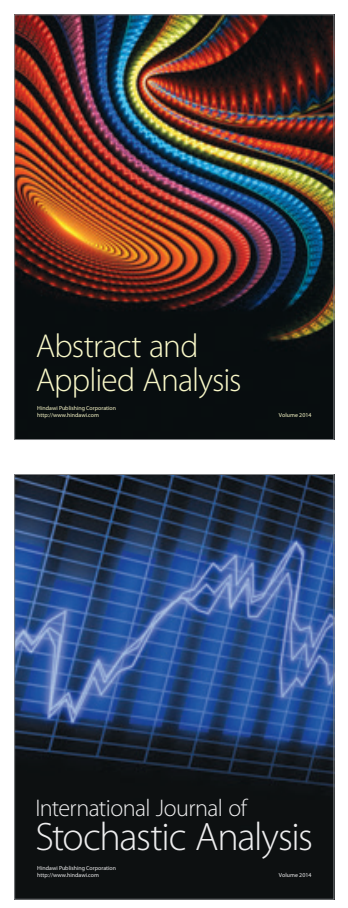

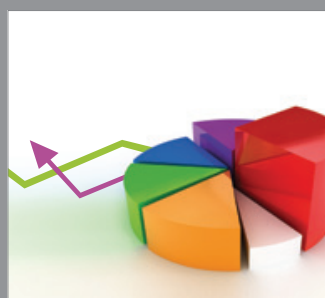

ournal of

Probability and Statistics

Promensencen
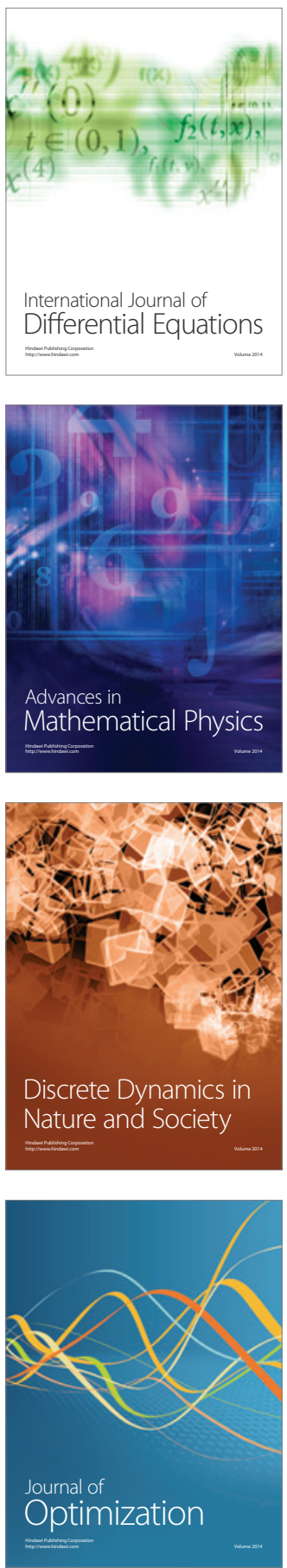